\title{
Perceptions of Consumers in the Airline Industry Using a Qualitative Data Analysis Methodology: An Applied Research under an International Orientation
}

\author{
David Rimbo $^{1}$, Rocky Nagoya ${ }^{1}$, Ikin Solihin ${ }^{1}$, Jorge Mongay ${ }^{2}$ \\ ${ }^{1}$ Executive Education, Universitas Pelita Harapan, Jakarta, Indonesia \\ ${ }^{2}$ ESIC Business and Marketing School, Madrid, Spain \\ Correspondence: Jorge Mongay, Avenida Valdenigrales s/n. Pozuelo de Alarcón, 28223 Madrid, Spain.
}

Received: February 23, 2017

Accepted: March 14, 2017

Online Published: March 30, 2017

doi:10.5539/ibr.v10n5p22

URL: https://doi.org/10.5539/ibr.v10n5p22

\begin{abstract}
Greater digital connectivity, improved practical functionalities of internet and website interface, and the wide embrace of the social-media lifestyle, have induced higher level of activism and participation by the consumer class, as evidenced by the more frequent posting of online customers' reviews. The textual and qualitative features of online customer reviews can be effectively reviewed and analyzed through the application of robust qualitative data mining methodology and text statistics. Qualitative Data analysis methodology (QDA) greatly helps with the technical steps for the codification, interpretative analysis, hypothesis testing, and predictive iterations. This research uses this methodology and proceeds with an in-depth analysis of $\mathrm{N}=1222$ written reviews to explain the most common complaints of passengers under an international and comparative approach of two airlines in Asia. The conclusions and results of the research are in line with other quantitative research done by (Messner \& Wolfang, 2016) stating that Food and Beverages, In-flight entertainment and the quality of the seats in the encounter stage of the service should be the major concerns of today's airlines.
\end{abstract}

Keywords: customer reviews, airline industry, qualitative data analysis, QDA, CAQDAS, qualitative research, experiential analysis

\section{Introduction}

\subsection{General Introduction}

In this study, QDA methodology has been applied in the retrieval, review and in depth analysis of textual data from Verified Reviews on Singapore Airlines (SQ) and Thai Airways (TG), respectively with a total of N=1222 reviews. This study aims to discover negative feelings, sentiments, personal viewpoints and experiences as communicated through the Skytrax Verified Reviews on SQ and TG, in order to derive meaningful insights as to the significant areas of concern of passengers, and the perceived shortcomings in product offerings and service delivery by the respective airlines, which could appropriately be considered by concerned parties for the devising of practical and targeted strategies, and improvement steps to uplift the overall flying experience of passengers.

\subsection{Relevant Scholarship}

Due to greater digital connectivity, improved user-friendliness and expanded functionalities, customers and consumers have opted more and more to provide their respective comments, inputs, reviews, and complaints, hence, the articulation of positive or negative sentiments and feelings regarding particular products, services, and customers handling, through online channels, may it be through the official websites, chat-rooms, and apps of the concerned corporations and providers or through alternative representations and advocacy and media channels, such as chat-rooms, forums, online surveys, etc. Given the predominantly textual and qualitative nature of such online customer reviews, qualitative data analysis methodology serves as a reliable and effective framework for deciphering the often complex and multifaceted feedbacks and inputs as provided. Qualitative data tend to have cyclic dynamic and potentially be built-upon circular referencing construction, thus, the insights derived thereof would complement the lineal positions as typically arisen from the analysis of quantitative data.

This study aims to analyze the applicability and efficacy of qualitative data mining through the application of 
CAQDAS (computer assisted qualitative data analysis software) in understanding online customer's reviews and feed-backs in the airline industry. Thus, the premise that, being able to practically decipher and understand the substance and relevance of customers' reviews, using qualitative data analysis methodology, would allow airlines to strategically and tactically implement action steps which would improve the overall level of satisfaction of flying customers.

The rapid increase of digital interconnectedness in recent times coupled with the wide adoption of various social media and peer-to-peer platforms for communications in the cyber space, have inevitably altered the perception of consumers as to the effective and practical formats and channels for: 1 . The articulation of one's viewpoints, feed-backs, inputs, and comments, 2. The sharing of relevant experiences, observations, sentiments, and feelings, and 3. The accessing to and targeting of the desired audience and the sphere and extent of potential influence thereof ("virality" in cyber parlance). Invariably such trends of digital communication would have reshaped consumers' and customers' preference as to the avenues and channels best perceived to maximize the impact of the provision of the respective comments, reviews, feed-backs, and complaints regarding a particular product, service, transaction or customer handling. The case in point is the increasing volume and frequency in recent years of customers' reviews as lodged into probably the most important global source, the Skytrax forum, with the accompanying greater extent and depth of reviews and commentaries concerning top-of-mind airlines and airports.

The observable increase in volumes and frequencies of consumers' and customers' comments and reviews as directly and indirectly posted and lodged to the targeted companies' websites and digital channels and/or to the relevant consumers' advocacy organizations and/or to the media organizations and/or to the jurisdictional regulatory bodies, points to the growing importance of the need to effectively understand and respond to such 'online' feed-backs and inputs.

Spradley (1980) defines QDA as the process of gathering, organizing, structuring and manipulating information collected by researchers with the goal to create relations, interpret, and obtain conclusions. Mongay (2016) postulates that, qualitative analysis is effective in analyzing the often hidden aspects of sentiments, personal positions, bias and individual experiences, as manifested in written texts, hence, enabling the exploration on the inherent motivations of the authors of the written reviews or commentaries.

Even in the most quantitatively-oriented and structurally complex world of finance, the application of qualitative data analysis methodology is not a new phenomenon. Gippel in 2013 argued that, approaching finance research deductively through statistical modeling of large-scale numerical data sets is currently considered common practice, hence, the relevance and applicability of quantitative research methods. Nevertheless, there are plentiful of cases supporting the applicability of qualitative research methodology in the very exacting world of finance, one of such applications which has proven to be so monumental is the ground-breaking study of dividend policy by Lintner in 1956, which began as a qualitative research study. The study's conclusions regarding dividend policy still hold true today, over 50 years since he published.

\section{Method}

The advent of digital interconnectedness and the proliferations of digital and electronic based communication and messaging interface, have not only provided greater access for the widest cross-section of the consumers base to communicate and articulate specific view points, feedbacks, and comments, but have also resulted in the significant increase of the volume and frequencies of such online comments as now could be posted and lodged electronically with significant ease. Accordingly, the traditional manual data mining, coding, and iterations of interpretative analysis as conventionally practiced in qualitative data analysis methodology would no longer be adequate to handle the big volume and high-frequency features of today's online comments. Computer aided qualitative data analysis software (CAQDAS) helps to overcome such technical challenge. Mackensen and Wille (1999) postulated that, "Computer-aided techniques for the management, coding, retrieval, and analysis of textual data are of increasing importance in social science test research. Computer systems especially designed for text analysis allow the researchers to handle and to systematically organize huge amount of textual data. They provide enhanced coding and retrieval techniques, and include various statistical tools for hypothesis testing."

The aforementioned viewpoint is further supported by Kaczynski, Salmona, Smith (2012), which strongly vouch for the reliability and practical application of CAQDAS.

QDAS significantly assist the codifications and analysis steps as undertaken by researchers, enabling more robust discovery of important categories of data, analysis potential overlaps, and assessment on the degree of relevance of the textual data, which as appropriately fed into the incorporated statistical functions in the software, 
allow for comprehensive analysis and extensive manipulative simulation of the captured texts and associated quantitative observations. It is to no surprise that, the corporate world has also been embracing the use of CAQDAS

\subsection{The Interests of Airlines in Understanding Customers' Reviews}

The research by Buzzell and Gale (1987) indicates that, the market perception of the quality of a company's products or services is the most important factor which affects a company's performance. This is even more profound in the airline sector, given the typically heightened travel experience, as eluded to in the earlier section. Kutulmusoglu et al. (2016) argues that, passengers select preferred airlines based on certain priorities and impact level in considering the expected level of service, and such priorities might differ according to the airline chosen. Accordingly, customers' specific viewpoints about onboard Food and Beverage, (F\&B) service, loyalty program, cabin conditions, may in varying degree affect the customers' overall expectations of the flying experience with a particular airline. Hence, being able to somehow measure and interpret such viewpoints as expressed by airline customers brings immense value for the positioning and possible improvement of the core competitive advantage of a particular airline.

Here lies the technical challenge as postulated by Chen and Chang (2005) that, frequently airlines measure their customers' perception of the services provided, without having sufficient knowledge about their customers' expectations. And furthermore Chen and Chang argued that, misreading or misevaluating customer expectations may create serious problems in airlines' resource allocation decisions. Accordingly, there is the impetus for applying a technically robust data mining methodology to understand the feelings and sentiments as expressed by airlines' customers about (i) the product and service as offered, (ii) the context in which a company offers such product and services, and (iii) the infrastructure that enables the transaction to take place, which accordingly to Rayport and Sviokla (1994) are the three basic elements of customer's perception of product and service value.

\subsection{Ensuring Effective and Consistent Textual Analysis through Coding Protocols.}

1,222 verified customers reviews, 675 on Singapore Airlines (SQ) and 547 on Thai Airways (TG), as lodged with Skytrax, were extracted and analyzed for the purpose of this paper. United Kingdom based airline consultancy firm, Skytrax, runs annual airline and airport reviews and ranking which have become the widely accepted benchmarks in the air travel industry. Its Airport Ranking and Airline Ranking started respectively in 1999 and 2001, and are based on methodical surveys -as updated regularly- and the analysis of customers' reviews as posted on its passenger's forum. Verified customers' reviews have undergone detailed authentication by Skytrax, including the required submission of copies of e-tickets, booking details or boarding passes, hence, evidencing real and actual travel experience as commented upon in the particular customers' reviews.

This research aims at effectively decoding and better understanding the negative feelings and sentiments as articulated by SQ's and TG's passengers in their respective customers' reviews with Skytrax. Ziethaml et al. (1996) states that, service managers should listen to their customers' feedback early in the transaction process, and should effectively and accurately respond to their identified needs. Any deeper understanding of the expression and articulations of feelings, sentiments and personal viewpoints which underpinned the customers' reviews, would provide enhanced insights as to the significant areas of concern of passengers, and the perceived shortcomings in product offerings and service delivery by the respective airlines. Hence, potentially contributing to the devising of practical and targeted strategies, and improvement steps to uplift the overall flying experience of passengers. Understanding customers attitudes and loyalties helps the devising of effective strategies for the acquisition and retention of customers. In this study we do not intend to investigate the foundational behavioral, demographic, socio-economic, and physiographic dimensions which may have impacted on the contextual constructions of the customers' reviews. The coding protocols are applied under an inductive approach.

\subsection{The Coding Process and List of Codes Obtained through the Inductive Approach}

The codes resulting from the QDA research applied in the reviews comments are as the following ones:

Ticketing \& Reservation

- Code 1. Quality of Call-Center’s service

- Code 2. Quality \& ease-of-use of on-line platform

- Code 3. Ease for making payment \& completing transaction

- Code 4. Ease for making seat reservation

- Code 5. Ease for amending reservations 
- Code 6. Options for booking menu

- Code 7. Options for special requests/assistance

Check-in \& Airport Handling

- Code 8. Meet \& greet hospitality

- Code 9. Quality \& standard of Check-in procedures

- Code 10. Efficiency of Check-in procedures

- Code 11. Helpfulness \& Attentiveness

- Code 12. Clear articulation of important logistics \& schedules

Pre-Boarding

Code 13. Quality \& Comfort of Airline Lounge Dedicated Lounge for Frequent Flyers. (Dedicated Lounge for Business Class, Dedicated Lounge for First Class, Quality of F\&B, Quality of Service by lounge staff, Quality of amenities \& reading materials, Quality of ambience \& cleanliness, Standard \& design of seating area).

Code 14. Quality \& comfort of Waiting Area (Gate-Front is more of a function of the overall standard of the Airport, not so much of the airline)

Code 15. Clarity of Pre-Boarding announcement

Code 16. Clarity of Boarding announcement

Code 17. Sequence of boarding

Code 18. Attentiveness on special assistance requirements

Code 19. On-time Departure

Boarding

Code 20. Greetings \& direction to seat

Code 21. Assistance with cabin luggage

Code 22. Pre-flight PA. (Flight-deck Crew, Cabin Crew)

Code 23. Safety PA \& Demonstration

In-flight Service

Code 24. Quality of service. (Friendliness, Helpfulness, Professional delivery of services)

Code 25. Responsiveness. (Attentiveness to special requests, Response time, Proactiveness in ensuring greater passengers comfort).

Code 26. Quality of F\&B Service. (Cabin crews' F\&B serving attire, Method of serving, Service attitude).

Code 27. Quality of F\&B. (Quality of ingredients, Presentation, Tastefulness \& flavors. Serving temperature, Selection and quality of food, Appetizers, Main course, Desserts, Cheese \& Fruits, Snacks \& Condiments, Options for special dietary requirements, Selection and quality of beverages, Welcome drinks, Alcoholic, Non-alcoholic, Chilled \& fresh, Hot beverages).

In-flight Comfort

Code 28. Overall quality of aircraft

Code 29. Cleanliness of cabin. (Cabin, Galley, Toilets, Overhead compartments).

Code 30. Lighting \& ambience setting. (Reading light, Ambience lighting)

Code 31. Pressurization \& humidification

Code 32. Quality of Seat. (Design \& overall quality of materials, Seat width, Seat pitch, Recline angle, Fully lie-flat bed seat).

Code 33. Seating configuration. (Aisle access, Legroom, Privacy).

Code 34. Storage Compartments

Code 35. Charging Ports

In-flight Amenities 
Code 36. Quality of in-flight kits by travel class

Code 37. Head support, pillows and duvets/blankets

Code 38. Reading materials

Code 39. In-flight entertainment IFE. (Audio and Video- AVOD Library \& Selection, Quality of head phones \& control panel, In-flight media publication).

Code 40. Toilet amenities.

Code 41. In-flight shopping. (Selection of products, Quality of in-flight sales service).

Arrival Preparation

Code 42. PA upon Aircraft Descent. (Flight-deck crew, Cabin crew)

Code 43. Cabin Preparation

Code 44. Safety Check \& PA

Disembarkation

Code 45. Arrival PA. (Airport information, Connecting flights information)

Code 46. Disembarkation Sequence \& Procedures

Code 47. Attentiveness to special/assistance requirements

Code 48. Hand-over to Ground crews Procedures

Arrival Services \& Ground-handling

Code 49. Ground-handling assistance

Code 50. Luggage collection assistance

Code 51. Lost \& Found assistance

Code 52. Others.

Throughout the coding process, the researchers discovered textual references which are unclear and not specific, hence, the difficulty in coding them into the established categories. Accordingly, a generic code labeled "Others' is created to accommodate these miscellaneous categories. Given the very low frequency of codes under this category, this approach was considered not likely to cause statistical significance bias.

Upon the second phase analysis of the results of the initial Coding of TG and SQ Customers' Reviews, the team of researchers observed potential overlaps of certain codes. Thus, further analysis and discussion among the authors ensued to determine the contextual justifications and practical considerations for the amalgamation of certain codes. Such codes merging exercise lead to the final 36 (thirty six) coding names.

\section{Results}

The findings show that complaints in the reviews relate to a total of 36 codes stated in the appendix 1 and 2 . It is important to state that the codes which appear more often are very similar in both companies, suggesting that customers pay attention to the same issues when they travel, despite the airline they use, or at least when comparing similar standard of airlines.

This section is presenting the Top 7 most important codes identified in both companies, due to the fact that the rest of the codes retrieved are highly fragmented. The research presents a concentration index of $\mathrm{C}_{7}^{62}$ in the case of Singapore Airlines, meaning that the top 7 most important complaints represent $62 \%$ of the total text retrieved when complaining in the reviews. In the study of Thai Airways, a concentration index of $\mathrm{C}_{7}^{58}$ appears stating that the top 7 complaints cover $58 \%$ of the total. 
SQ Main findings

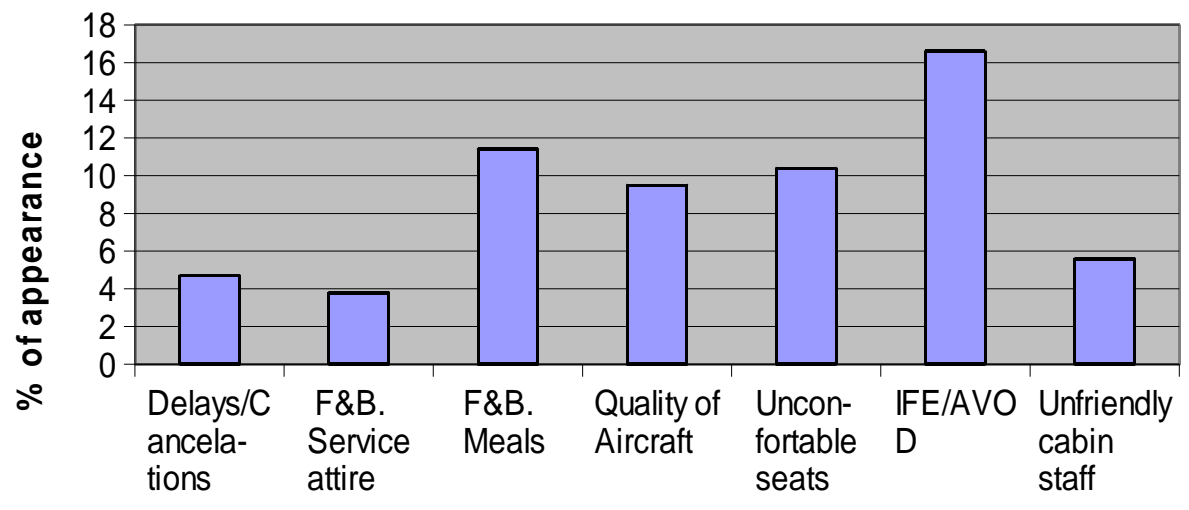

\section{Codes retrieved}

Figure 1. Results derived from main SQ findings

\section{TG Main findings}

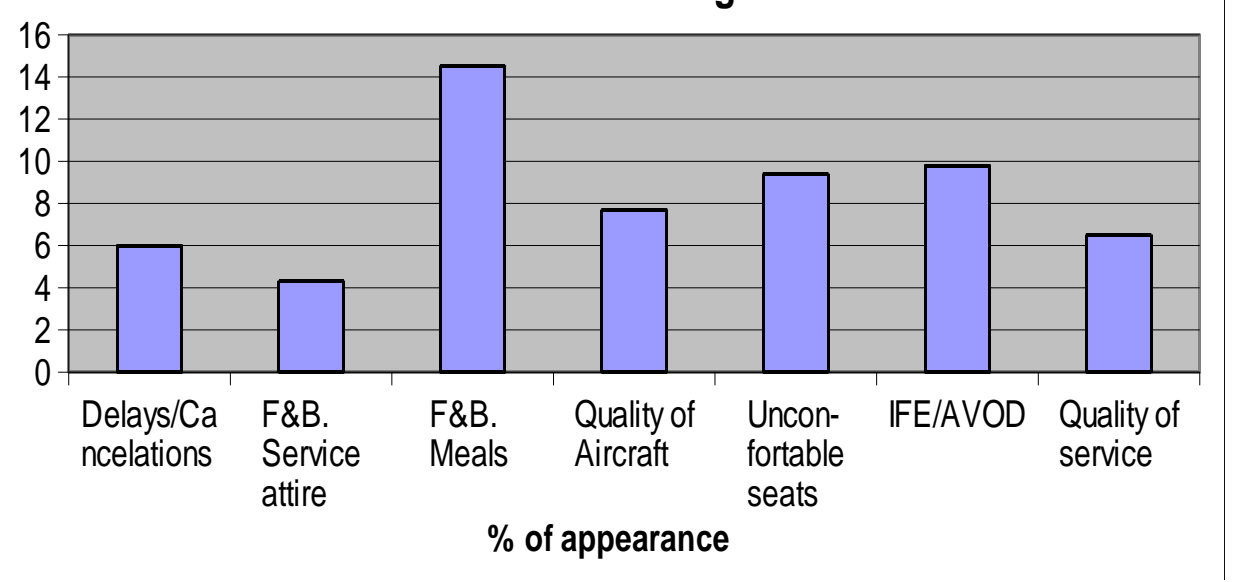

Figure 2. TG main findings

Table 1. Summary of main complaints expressed and retrieved using QDA methodology

\begin{tabular}{lll}
\hline Concept & Thai Airways (TG) & Singapore Airlines (SQ) \\
\hline Food and Beverages (F\&B). Meals & $14.5 \%$ & $11.40 \%$ \\
Food and Beverages (F\&B). Service attire. & $4.3 \%$ & $3.8 \%$ \\
Delays and cancellations & $6 \%$ & $4.7 \%$ \\
Quality of aircraft & $7.7 \%$ & $9.5 \%$ \\
Uncomfortable seats & $9.4 \%$ & $10.4 \%$ \\
IFE (In flight Entertainment) AVOD & $9.8 \%$ & $16 \%$ \\
Unfriendly Staff in Cabin &.$--- \%$ & $5.6 \%$ \\
Quality of Service & $6.5 \%$ & ------------- \\
\hline
\end{tabular}

\section{Discussion on limitations of the research}

The research explores in depth a total of 1222 reviews of complains from consumer's perspectives. Authors believe that this research can be improved by an adding a bigger number of airlines and related them to a geographic area. This step would help make the sample more reliable and would extent the research to other airlines making the comparative process more comprehensive. The written reviews appear in an internet portal which is really valuable in order to understand honesty in relation to feelings due that spontaneous comments are very valuable, according to the findings of Berg (2004) from a qualitative point of view. Still it is really difficult to guarantee or demonstrate that these are common problems to all airlines worlwide. No research has been applied comparing low cost with regular airlines, being these last two the target of this research. In the future, it would be required a wider perspective of this research and to extend the same research protocols to other companies in order to go beyond this particular case study in Thai Airways and Singapore Airlines. 


\section{Acknowledgments}

Thank you so much to Professor Dr. Gracia Ugut from Universitas Pelita Harapan (UPH) Executive Education, for giving us the opportunity to develop this piece of work under a perfect environment.

\section{References}

Berg, B. L. (2004). Qualitative Research Methods for the Social Sciences, Fifth Edition. Pearson Education, Inc.

Buzzell \& Gale (1987). The Pims Principles. Linking Strategy to Performance. The Free Press. New York.

Gippel, J. (2015). Masters of the Universe: What top finance academics say about the 'state of the field'. Australian Journal of Management, 40, 538-566. https://doi.org/10.1177/0312896215584450

Kaczynski, D., Salmona, M., \& Smith, T. (2014). Qualitative research in finance. Australian Journal of Management, 39(I) 127-135. https://doi.org/10.1177/0312896212469611

Lintner, J. (1956). Distribution of Incomes of Corporations Among Dividens, Retained Earnings, and Taxes. The American Economic Review, 46(2), Papers and Proceedings of the Sixty-eighth Annual Meeting of the American Economic Association. (May, 1956), 97-113.

Mackensen, K., \& Wille, U. (1999). Qualitative Text Analysis Supported by Conceptual Data Systems. Quality \& Quantity: International Journal of Methodology, 33(2), 135-156. https://doi.org/10.1023/A:1004305723553

Messner, W. (2016). The impact of an aircraft's service environment on perceptions of in-flight food quality. Journal of Air Transport Management, 53, 123-130. https://doi.org/10.1016/j.jairtraman.2016.02.010

Mongay, J. (2016). Perceptions of consumers in the financial industry under A Qualitative Data Analysis methodology. International Journal of Trade and Global Markets, 9(1), 33-44. https://doi.org/10.1504/IJTGM.2016.074136

Rayport \& Sviokla (1994). Managing the Market Space. Harvard Business Review. Nov-Dec issue. Retrieved from https://hbr.org/1994/11/managing-in-the-marketspace

Spradley, J. P. (1980). Participant Observation. Rinehart \& Winston, New York.

\section{Appendix A: Results Derived from Thai Airways Analysis}

\begin{tabular}{|l|l|c|c|c|}
\hline Category & Code & Count & $\%$ Codes & \% Cases \\
\hline Advertisement & Misleading Advertising & 1 & $0.10 \%$ & $14.30 \%$ \\
\hline Reservation & Seats Reservation Problem & 5 & $0.50 \%$ & $42.90 \%$ \\
\hline Payment & Difficulties for Making Payment \& Completing Transaction & 1 & $0.10 \%$ & $14.30 \%$ \\
\hline Payment & Less Value for Money & 12 & $1.30 \%$ & $71.40 \%$ \\
\hline Payment & Poor Compensation for Bad Service & 2 & $0.20 \%$ & $14.30 \%$ \\
\hline Check-in & Check-in Issues & 16 & $1.70 \%$ & $85.70 \%$ \\
\hline Check-in & Unhelpful and Inattentive Check-In Staffs & 12 & $1.30 \%$ & $71.40 \%$ \\
\hline Lounge & Below Expectation for Airline Lounge & 25 & $2.70 \%$ & $71.40 \%$ \\
\hline Boarding & Problems with Boarding & 8 & $0.90 \%$ & $57.10 \%$ \\
\hline
\end{tabular}

\section{Appendix B: Results Derived from Singapore Airlines Analysis}

\begin{tabular}{|c|c|c|c|c|c|}
\hline Category & Code & Count & $\%$ Codes & Cases & $\%$ Cases \\
\hline Ticketing \& Reservation & Difficulties for making payment $\&$ completing transaction & 2 & $0.30 \%$ & 1 & $14.30 \%$ \\
\hline Ticketing \& Reservation & Seat Reservation Problem & 8 & $1.00 \%$ & 5 & $71.40 \%$ \\
\hline Check-in \& Airport Handling & Check in Issues & 5 & $0.60 \%$ & 3 & $42.90 \%$ \\
\hline Check-in \& Airport Handling & UnHelpful \& InAttentive Check-in Staffs & 4 & $0.50 \%$ & 3 & $42.90 \%$ \\
\hline Pre-boarding & Below Expectation for Airline Lounge & 7 & $0.90 \%$ & 4 & $57.10 \%$ \\
\hline Pre-boarding & Problems with Boarding & 5 & $0.60 \%$ & 3 & $42.90 \%$ \\
\hline Pre-boarding & Delayed and Canceled Flights Problems & 37 & $4.70 \%$ & 7 & $100.00 \%$ \\
\hline In-flight Service & Quality of service is under expectation & 3 & $0.40 \%$ & 3 & $42.90 \%$ \\
\hline In-flight Service & Quality of F\&B Service is under expectation & 30 & $3.80 \%$ & 7 & $100.00 \%$ \\
\hline
\end{tabular}

\section{Copyrights}

Copyright for this article is retained by the author(s), with first publication rights granted to the journal.

This is an open-access article distributed under the terms and conditions of the Creative Commons Attribution license (http://creativecommons.org/licenses/by/4.0/). 